\title{
Ultrasound Elastography in Musculoskeletal Radiology: Past, Present, and Future
}

\author{
Žiga Snoj, MD, PhD ${ }^{1,2} \quad$ C. H. Wu, MD 3 M.S. Taljanovic, MD ${ }^{4} \quad$ I. Dumić-Čule, MD \\ E. E. Drakonaki, $M^{6}$ Andrea S. Klauser, $M^{7}$
}

${ }^{1}$ Radiology Institute, University Medical Centre Ljubljana, Ljubljana, Slovenia

2 Faculty of Medicine, University of Ljubljana, Ljubljana, Slovenia

${ }^{3}$ Department of Physical Medicine and Rehabilitation, National Taiwan University Hospital College of Medicine, National Taiwan University, Taipei, Taiwan

${ }^{4}$ Department of Medical Imaging, University of Arizona, Business, SimonMed Imaging, Scottsdale, Arizona

${ }^{5}$ Department of Diagnostic and Interventional Radiology, University Hospital, Zagreb, Croatia

6 Medical School of the European University, Cyprus

${ }^{7}$ Department of Radiology, Division of Rheumatology and Sports

Imaging, Medical University Innsbruck, Innsbruck, Austria

Address for correspondence Žiga Snoj, MD, PhD, Radiology Institute, University Medical Centre Ljubljana, Ljubljana, Slovenia (e-mail: ziga.snoj@gmail.com).

Semin Musculoskelet Radiol 2020;24:156-166.

\begin{abstract}
Keywords

- ultrasound

- elastography

- musculoskeletal

- quantitative imaging

- shear deformation

Ultrasound elastography (USE) is becoming an important adjunct tool in the evaluation of various musculoskeletal (MSK) traumatic conditions and diseases, with an increasing number of applications and publications in recent years. This rapidly evolving technique enhances the conventional ultrasound (US) examination by providing information on the elastic properties of tissue alongside the morphological and vascular information obtained from B-mode US and Doppler imaging. Those performing USE must have basic knowledge of its proper imaging techniques and limitations. In this review article, we place the USE in historical perspective and discuss basic techniques and current applications of USE in the evaluation of various traumatic and pathologic conditions of fasciae, nerves, muscles, tendons, ligaments, and MSK soft tissue masses.
\end{abstract}

Ultrasound elastography (USE) is a method that evaluates tissue elasticity in real time during ultrasound (US) imaging. ${ }^{1,2}$ It has added a new dimension to US and is considered the most important advance in US technology since Doppler imaging implementation. ${ }^{1,2}$ The technique has a wide range of applications in radiology including musculoskeletal (MSK) soft tissue imaging. ${ }^{1,2}$ This article reviews the history and physics of this technique, focuses on current applications in the assessment of muscle, tendon, nerve, ligaments, and soft tissue masses, and discusses future potential applications and technical considerations.

\section{The Past}

Palpation is a qualitative diagnostic technique that has been used in medical practice for thousands of years. Hippocrates was reported to use palpation for battle injuries, identifying that if the bone is not visible, it can assist in locating the weapon mark, and, for head injuries, it can determine whether the cranium beneath the flesh is strong or weak. ${ }^{3}$ Palpation is still used today and is an essential component of any clinical evaluation. However, palpation is based on subjective evaluation with limited disease detection and limited organ accessibility. ${ }^{3}$ 
Elastography is a field in science that aims to overcome these limitations by providing noninvasive imaging of tissue mechanical properties. The US evolution has come to the point where real-time elastography is possible and now integrated in commercially available US machines. Along the path of the evolution of US, many important steps need to be noted as the foundation for modern MSK radiology. The first report on MSK sonography was published in 1958. ${ }^{4}$ In 1972, the first B-scan image of a human joint was published describing the use of US imaging in differentiating Baker's cysts from thrombophlebitis. ${ }^{4}$ The first report of using US-guided joint aspiration was published in 1981 in a septic arthritis work-up. ${ }^{4}$ In 1994, the first application of power Doppler was reported with demonstration of soft tissue hyperemia in MSK disease. ${ }^{4}$ Contemporary elastography was introduced in the imaging field, with applications in magnetic resonance imaging (MRI) and US. ${ }^{5}$ In the beginning USE applications were in vitro, and they subsequently evolved into a real-time tool for in vivo imaging of the distribution of tissue strain and elastic modulus. Over time US has become an important cornerstone of MSK imaging with USE striving to find its place in everyday clinical practice. ${ }^{4}$

\section{The Present}

USE has developed rapidly in recent years with an everincreasing number of publications, mainly due to its wide availability on commercial US systems. ${ }^{3}$ The European Society of Skeletal Radiology published a group consensus, based on reviewing the literature on USE MSK applications, stating that USE currently has a low level of indication in clinical practice but acknowledging it is a promising technique for soft tissue masses and nerve entrapment. ${ }^{6}$ Radiologists performing MSK USE must have basic knowledge of the physics and limitations of the USE techniques used in various tissues. Depending on the method of force application and data processing, several USE methods are available. ${ }^{3}$ In the field of MSK radiology, compression elastography (CE) and shear wave elastography (SWE) are the most commonly used methods.

\section{Compression Elastography}

The principle exploited in $\mathrm{CE}$ is that the compression of tissue produces displacement, or strain. ${ }^{3}$ In stiffer tissue, the strain is minimal; in softer tissue, the strain is greater. Tissue displacement is calculated from repeated manual compression by using a handheld US transducer. ${ }^{3}$ The displacement distribution is shown in an elastogram, usually displayed as a color-coded image superimposed over the B-mode image. ${ }^{7}$ Red is typically used to depict softer tissue, blue harder tissue, and yellow or green to depict intermediate elasticity. ${ }^{7}$ It is notable that the color indicates the relative stiffness of the tissues, and therefore the color coding can be modified. ${ }^{7,8}$ Reliability of CE is another challenge because too heavy or too gentle compression can distort elastograms due to nonlinear changes in tissue elasticity. Most vendors provide the amount of manual compression as a visual indicator on the screen. ${ }^{7}$ For example, a "quality factor" $>60$ indicates the optimal compression force, whereas a "strain indicator" indicates if displacement is sufficient to calculate local strains within the region of interest (ROI). ${ }^{9}$ In some systems, semiquantitative tools are provided to analyze image characteristics as a strain ratio. ${ }^{3}$ This is an index of relative elasticity of target ROI versus reference ROI (usually the subcutaneous fat layer).,

\section{Shear Wave Elastography}

A principle exploited in SWE is that directional force leads to tissue shear deformation propagating as a shear wave. ${ }^{3}$ SWE is a type of dynamic elastography and based on the measurement of the tissue propagation velocity distribution of the directional shear wave, produced by the US pulse. ${ }^{3,7}$ In addition to qualitative depiction with elastograms, SWE can also quantify the absolute elasticity value of an imaged structure. ${ }^{3}$ Shear waves move at a faster rate through harder tissue. The quantitative measurement can be expressed as shear wave velocity in meters per second $(\mathrm{m} / \mathrm{sec})$ or as tissue elasticity based on shear modulus calculation in kilopascals $(\mathrm{kPa}){ }^{3,10}$

\section{Terminology in Elastography}

Several physical misconceptions and nomenclatorial inaccuracies in the literature abound when reporting the elastography results. ${ }^{10}$ Stiffness, shear modulus, and shear wave velocity are distinct physical phenomena and should not be used interchangeably. ${ }^{10}$ Thus when reporting elastography results in $\mathrm{kPa}$, the term shear modulus should be used, and when reporting elastography results in $\mathrm{m} / \mathrm{sec}$, the term shear wave velocity should be used. ${ }^{10}$

\section{Fascia}

Interest in fascia has grown in the recent years and is a subject of an increasing number of publications. Elastography research has mostly been conducted on plantar fascia; reports of other fascia locations are scarce. ${ }^{11-13}$ This is understandable because plantar fasciitis is a common pathology and frequently imaged in clinical practice. ${ }^{11}$ Patients with plantar fasciitis show significantly softer plantar fascia compared with healthy individuals, even in those with typical clinical manifestations of plantar fasciitis but without abnormalities on B-mode sonography (-Fig. 1). ${ }^{14-16}$ The experience with other fasciae is limited, with only a few articles reporting data on thoracolumbar fascia and perimuscular fascia of the posterior thigh and calf. ${ }^{12,13}$ Langevin et al showed that the shear strain of thoracolumbar fascia was $\sim 20 \%$ lower in human subjects with chronic low back pain compared with a control group. ${ }^{12}$ It was shown that USE can depict the effects of manual therapy and thus expand the utility of elastography in fascia evaluation beyond purely diagnostic purposes. ${ }^{13}$

\section{Nerves}

Most nerve USE studies have focused on the median nerve at the wrist. ${ }^{17}$ This is most likely because the median nerve runs superficially with good depiction on US, and carpal tunnel syndrome (CTS) is a common pathology (-Fig. 2). ${ }^{17}$ It was 


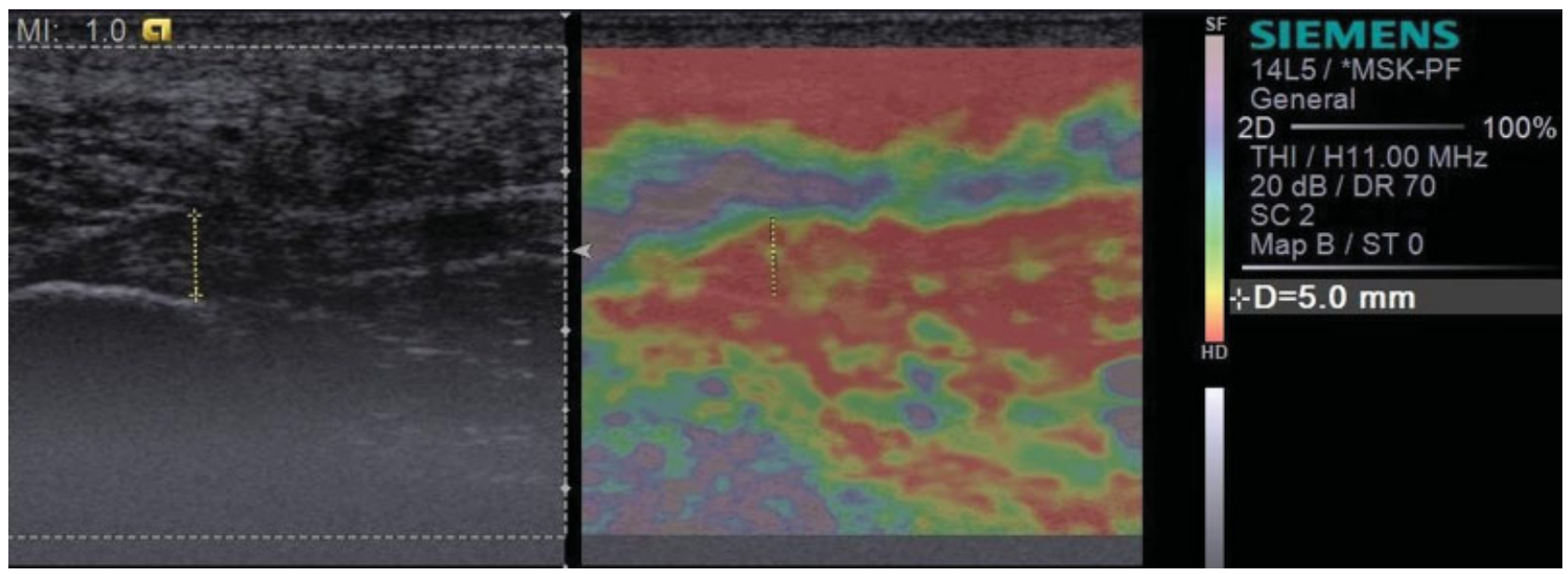

Fig. 1 B-mode image and compression elastogram (CE) of the plantar fascia in a 51-year-old man with plantar fasciitis. The scattered areas of yellow color in the red plantar fascia indicate relative tissue softening. The images were acquired with Siemens $\mathrm{CE}$ where red indicates hard tissue and blue-purple indicates soft tissue.

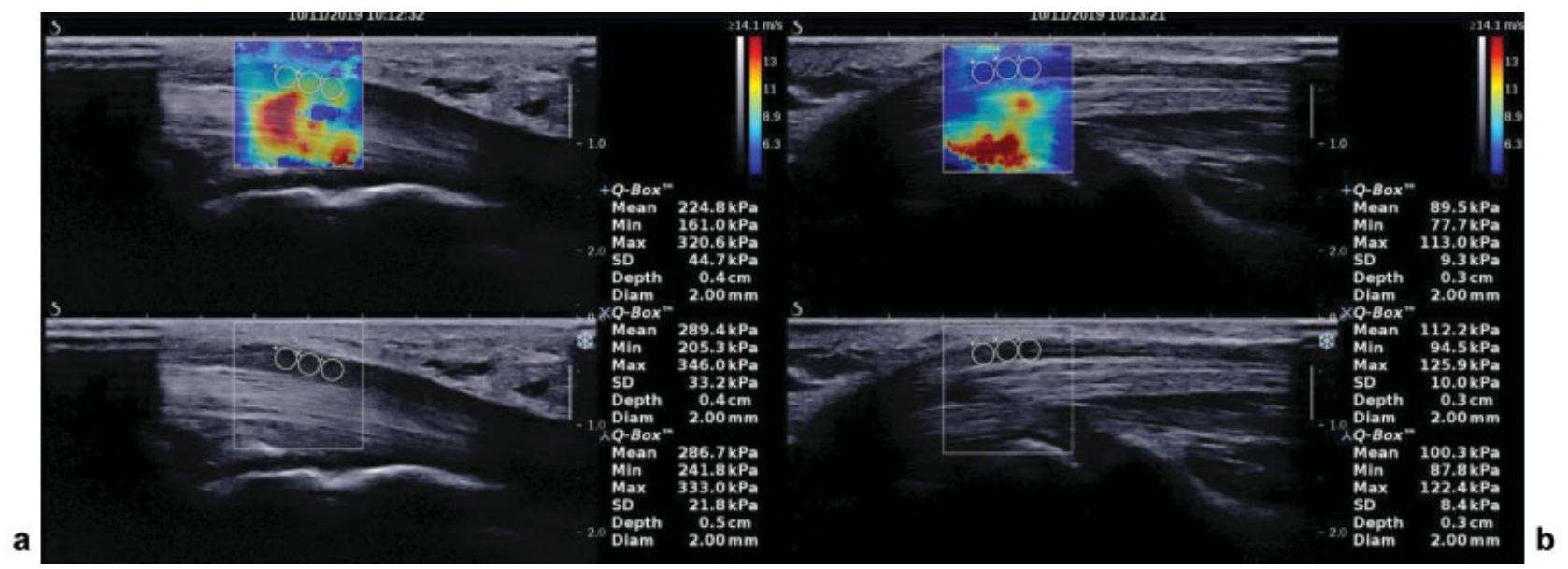

Fig. 2 Longitudinal elastogram and B-mode image at the carpal tunnel of both forearms in a patient diagnosed with right-sided carpal tunnel syndrome (CTS). Panel A: A thickened median nerve imaged longitudinally in the right forearm; CTS was graded as moderate on electromyelogram with measured cross-sectional area $18 \mathrm{~mm}^{2}$ on US at the carpal tunnel inlet. Panel B: Left forearm of the same patient with no complaints suspicious of CTS. Shear wave elastography with scale depicting shear wave speed; the values of regions of interest are expressed in shear modulus.

shown in healthy individuals that the median nerve is stiffer at the wrist than in the forearm. ${ }^{18}$ Furthermore, the two sides do not differ significantly; thereby a contralateral median nerve may serve as an internal control. ${ }^{18}$ Most of the studies on CTS showed an important difference in median nerve stiffness between patients with CTS and healthy controls. ${ }^{17}$ Subsequently, various cutoff values for the diagnosis of CTS were proposed. ${ }^{17}$ Above the proposed cutoff values is the ratio between the median nerve stiffness at the carpal tunnel versus the median nerve stiffness in the forearm. ${ }^{19}$ A cutoff ratio of 1.48 was proposed for the diagnosis of CTS, yielding a sensitivity of $97.7 \%$ and a specificity of $100 \%{ }^{17,19}$ Nerve USE improves the diagnostic accuracy of US examination in combination with conventional B-mode US and aids in sonographic grading of carpal tunnel severity. ${ }^{17}$ It was shown that after carpal tunnel release or corticosteroid injection, median nerve stiffness decreases significantly. ${ }^{20,21}$ Moreover, it was reported that nerve USE may be a more sensitive measure of nerve recovery than the cross-sectional area. ${ }^{22}$ With the increasing number of image-guided interventional procedures of the MSK system, the use of USE may become an important modality in the follow-up period. ${ }^{23-25}$

In other locations, nerve USE has been used in the evaluation of ulnar neuropathy, brachial plexus, and peripheral neuropathy. ${ }^{17,22}$ Similar to the median nerve in CTS, increased ulnar nerve stiffness was shown in ulnar neuropathy. However, no relationship between the severity of ulnar neuropathy and USE was shown. ${ }^{17}$ Marked increase in stiffness in the irradiated brachial plexus in comparison with the unirradiated contralateral plexus in patients with breast cancer after radiotherapy was reported. ${ }^{17}$ USE showed promising results in the evaluation of peripheral neuropathy with detecting subclinical diabetic polyneuropathy that is not evident on standard electrodiagnostic studies. ${ }^{17,22}$

\section{Soft Tissue Masses}

USE has been extensively explored in tumors outside the MSK system and was found useful in differentiating benign and malignant lesions mainly in the breast. ${ }^{26}$ However, the 


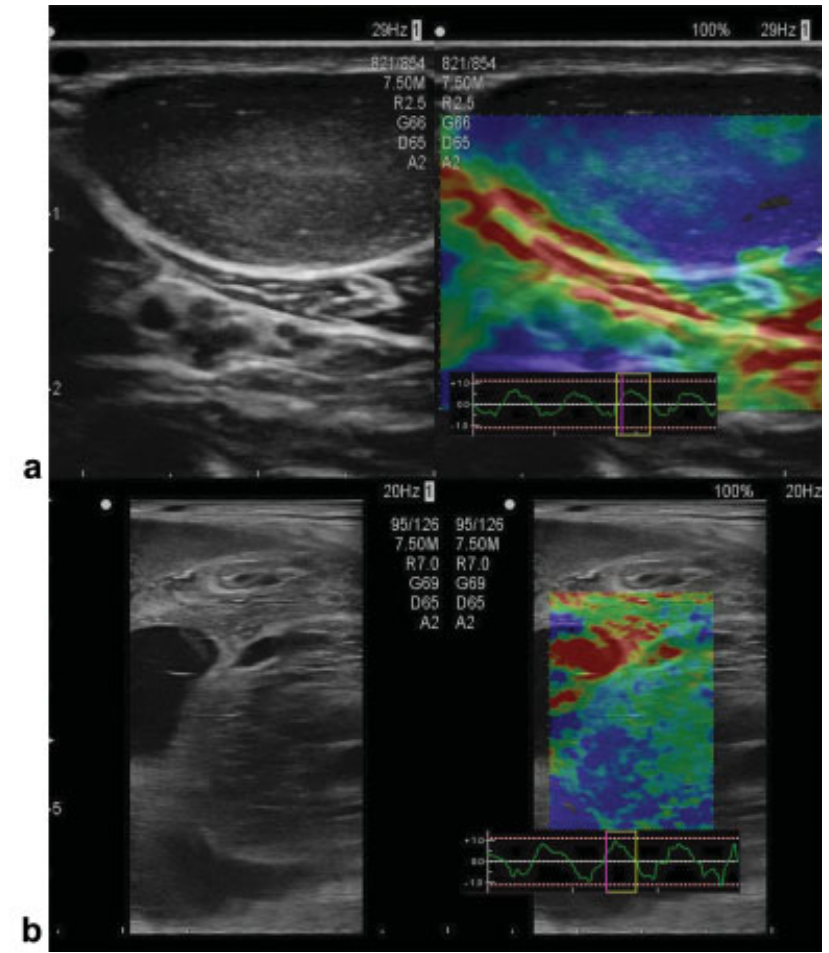

Fig. 3 B-mode and elastogram image of the forearm and the upper thigh in a patient with neurofibromatosis type 1 . Panel a: In the patient's forearm, a well-defined homogeneously hypoechoic subcutaneous lesion is seen with the corresponding elastogram showing a homogeneously hard lesion. Panel b: In the patient's upper thigh, a well-defined heterogeneous lesion is seen with corresponding elastogram showing heterogeneous tissue morphology. Red regions represent soft cystic areas; blue regions represent hard regions of tumorous soft tissue. The compression cycles may be appreciated.

value of USE in the differentiation of MSK tumors is not straightforward because a much greater variation in grade and histologic types occurs in sarcomas compared with breast lesions. ${ }^{26-29}$ One of the early articles on this topic was a short pictorial essay by Lalitha et al. They briefly reported that hemangiomas and neurofibromas demonstrate soft to firm consistency, typical of benign lesions (-Fig. 3). ${ }^{30}$ Further studies supported these findings with the exception of a study by Pass et al that questions the ability of USE to detect malignancy over B-mode consensus classification. ${ }^{27-29}$

The value of USE for differentiating malignant from benign lesions is supported by a study including 73 histologically proven soft tissue masses that showed higher stiffness of malignant versus benign lesions. ${ }^{27}$ Similarly, Riishede et al examined 61 histologically proven soft tissue masses with $\mathrm{CE}$ and showed that strain ratios of malignant tumors were significantly higher compared with strain ratios of benign tumors but with overlapping confidence intervals. ${ }^{29}$ They proposed that differentiation between malignant and benign tumors may be achieved by $\mathrm{CE}$, a finding that may potentially reduce the number of histologic biopsies. ${ }^{29}$ However, contrary to other studies, the largest published study with 105 biopsy-proven MSK soft tissue tumors found no difference in SWE values between benign and malignant lesions. ${ }^{28}$ Inter-

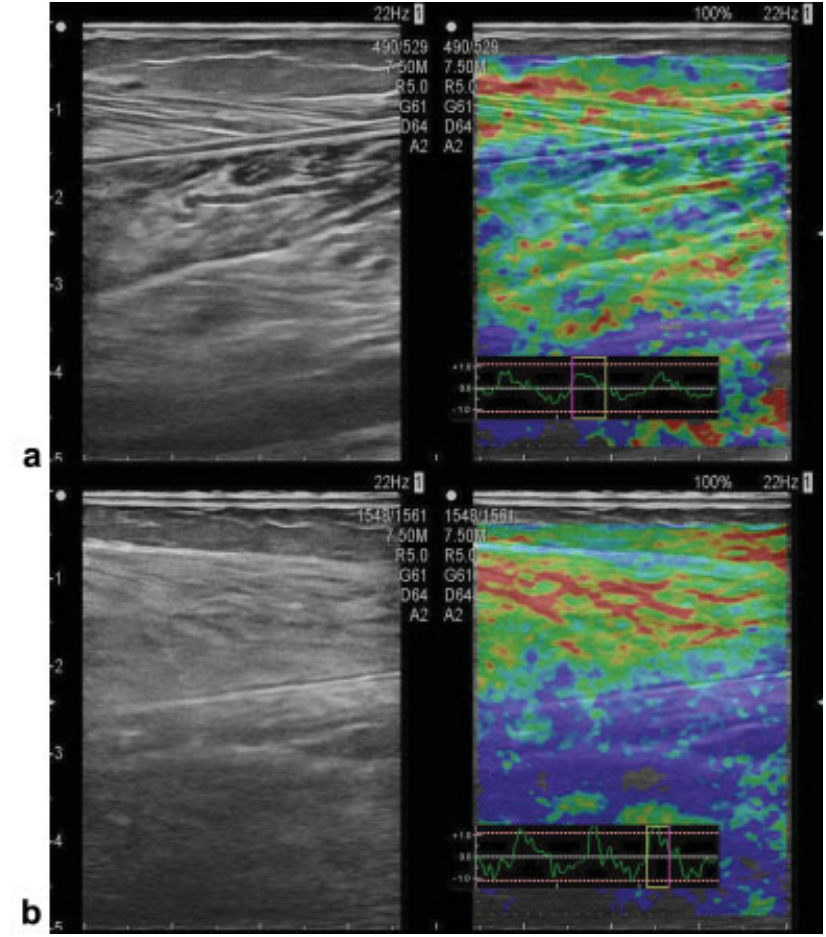

Fig. 4 Bilateral B-mode and elastogram image of the medial head of gastrocnemius and soleus musculature in the distal third of the lower leg in a patient with complaints of pain in the left calf). Panel a: A longitudinal US image of the patient's right lower leg showing preserved muscle structure with predominantly intermediate stiffness on elastogram. Panel b: A longitudinal image showing hyperechoic muscle tissue with loss of muscular structure and elastogram showing predominantly soft stiffness of the medial head of gastrocnemius muscle and predominantly hard stiffness of the soleus muscle.

estingly, a 2018 article explored the use of USE beyond soft tissue tumors by reporting a case series of bone tumors in children. ${ }^{31}$ The authors reported that most malignant bone tumors were very stiff. ${ }^{31}$

\section{Muscle}

The use of USE to study muscles has been expanding in recent years. This trend may be attributed to increased research interest in sarcopenia as well as to a broad application in various disorders presenting with muscle pathology. ${ }^{32} \mathrm{~A}$ wide range of normal and abnormal muscle USE appearances are reported, without established reference values. ${ }^{33}$ However, contralateral muscle proved to serve as a good internal control because bilateral muscle assessment did not show significant differences in muscle stiffness (-Fig. 4). ${ }^{34}$ Several studies showed greater stiffness of contracted muscle compared with the relaxed muscle (- Fig. 5). ${ }^{33}$ Gender differences were reported in lumbar musculature with male muscles proving to have higher stiffness; however, no gender differences were observed in the evaluation of calf muscles. ${ }^{35-37}$ There is no consensus in the literature on the effect of age on muscle stiffness, with some authors reporting increasing and others decreasing muscle stiffness. ${ }^{38-40} \mathrm{~A}$ few important factors may be debated regarding this disagreement. First, 


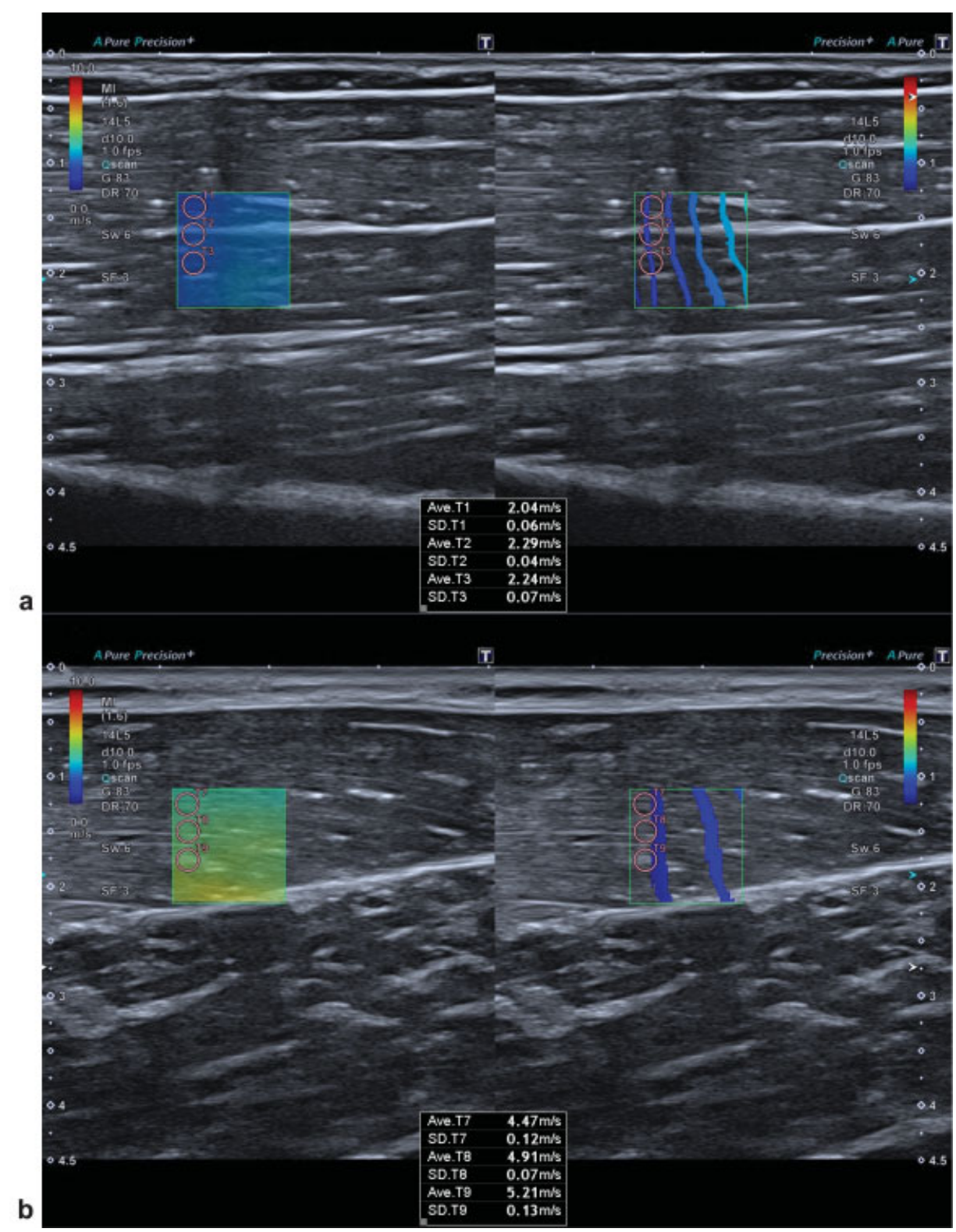

Fig. 5 B-mode image with elastogram overlay of the biceps brachii muscle in a healthy volunteer. A longitudinal image of a relaxed muscle (panel a) and flexed muscle (panel b). The images were acquired with shear wave elastography with the left box representing a color-coded elastogram and the right box representing the quality of a shear wave. The regions of interest (ROIs) should be placed in the box where the waves are most parallel. Values of ROls are expressed as shear wave velocity.

the difference in the evaluation protocol resulted in varied USE values based on the type of functional muscle assessment. ${ }^{23}$ Muscle stretching is responsible for a linear increase in muscle stiffness, whose magnitude depends on the type of muscle, joint stiffness, and positioning. ${ }^{33}$ Second, the selection of muscle groups across studies would influence USE values because certain muscle groups may not show the same aging pattern according to their function (e.g., axial versus appendicular muscles).

Yanagisawa et al demonstrated greater stiffness in the biceps brachii muscle of post- versus pre-exercise USE values. ${ }^{41}$ Different manual therapy procedures and muscle manipulation induce a change in muscle stiffness. ${ }^{33} \mathrm{~A}$ temporary increase of muscle stiffness was also shown with SWE in delayed-onset muscle soreness. ${ }^{42}$ These studies suggest the possibility of monitoring physiologic changes and broadening USE utility beyond pathologic processes. USE may prove useful in the evaluation of myofascial pain syndrome and in a rehabilitation process by depiction of myofascial trigger points..$^{43}$ In a pictorial essay, Botar et al demonstrated the use of USE in muscular trauma by depiction of alterations in elastograms by intramuscular hemorrhage and fibrosis. ${ }^{44}$ This indicates that our understanding of muscle injuries and their healing process may be broadened with USE. In studying muscle crush injuries in rabbit models with SWE, a significant difference was observed in Young's modulus with values significantly higher in the injured leg at 72 hours after crushing pressure in comparison with the uninjured leg. 45

The use of USE in various neuromuscular disorders has been studied ( - Figs. 6 and 7). ${ }^{46-48}$ Botar et al showed a 


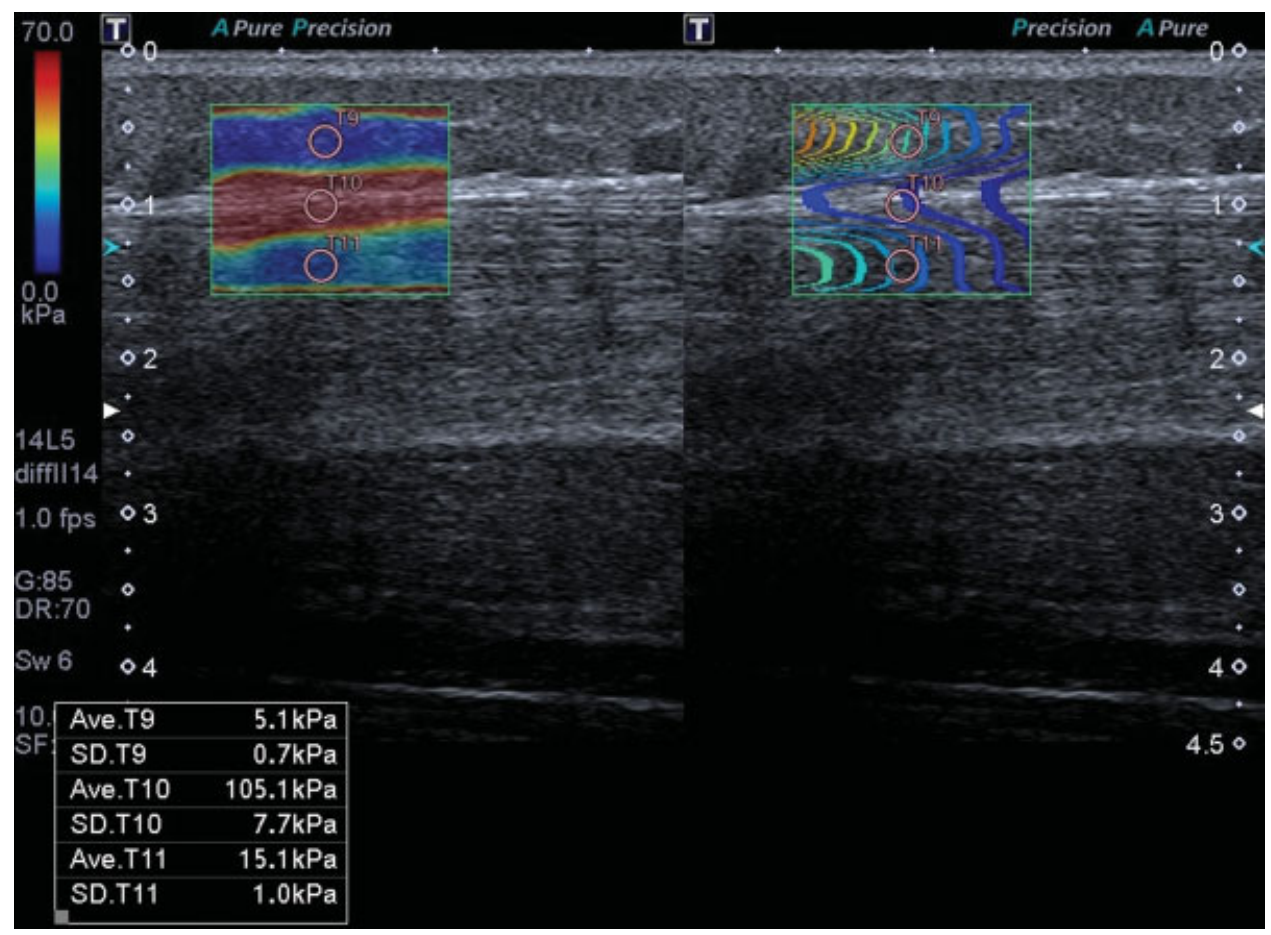

Fig. 6 Shear wave elastogram of the calf in a 12-year-old boy with Pompe disease. It is clearly depicted that the shear modulus is $5.1 \mathrm{kPa}$, $105.1 \mathrm{kPa}$, and $15.1 \mathrm{kPa}$ in the subcutaneous layer, the fascia, and the gastrocnemius muscle, respectively. The images were acquired with shear wave elastography on an Aplio i800 machine with the left box representing a color-coded elastogram and the right box representing the quality of a shear wave. The regions of interest (ROIs) should be placed in the box where the waves are most parallel. Values of ROIs are expressed as shear wave speed.

strong case for USE utility in inflammatory myopathies by direct correlation of SE muscle values and specific muscle inflammatory markers. ${ }^{47}$ SWE showed that the muscles are significantly stiffer on the poststroke spastic side in acute or chronic poststroke stage. ${ }^{48}$ Similar results were shown in patients with multiple sclerosis and cerebral palsy. ${ }^{47,49}$ Thus it may be concluded that USE may prove useful in patients with neuromuscular disorders with the potential of helping in the disease diagnosis pathway as well as monitoring the course of the disease and can serve as guidance for choosing the appropriate treatment. ${ }^{47}$ However, several factors are important when imaging muscle, and the methodology of USE in paretic muscle should be clarified. ${ }^{50,51}$

\section{Tendon}

Within the USE field of examining tendons, the Achilles tendon has received the most attention due to its ease of

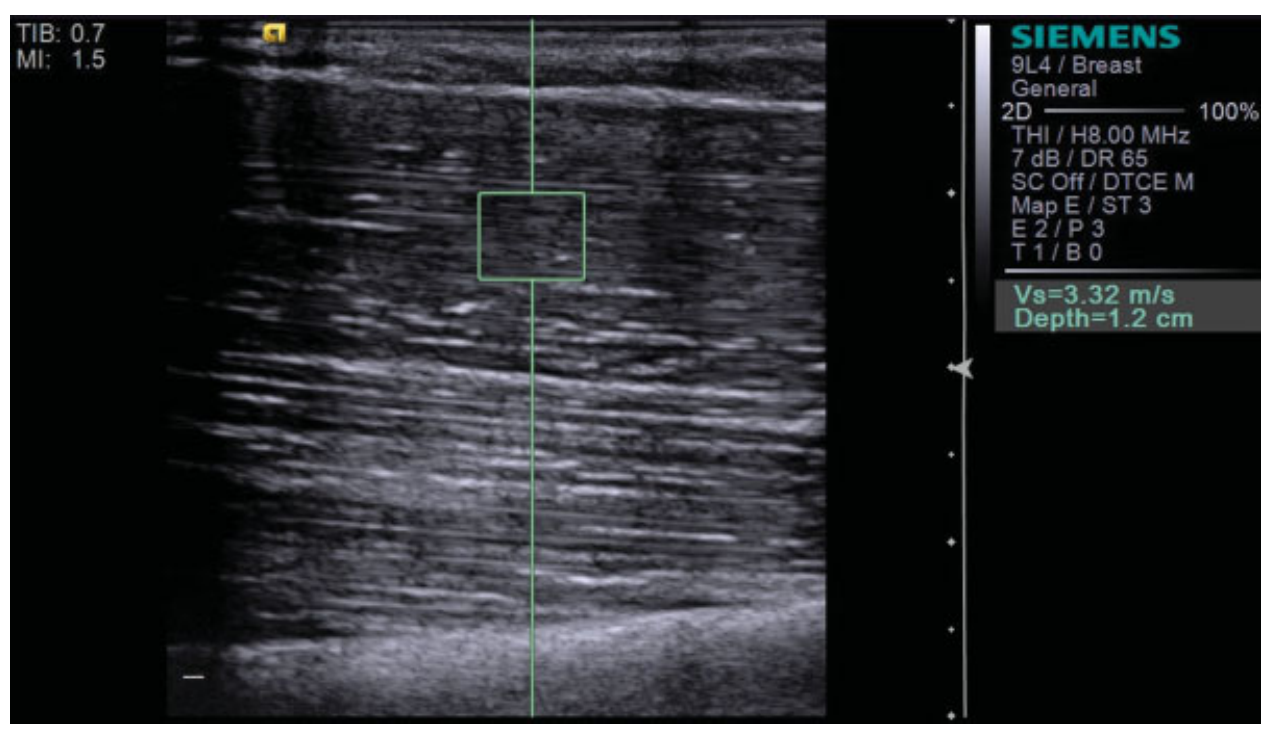

Fig. 7 Shear wave elastogram of the spastic biceps brachii muscle in a 34-year-old man with a left putaminal hemorrhage. The shear wave velocity within the region of interest was $3.32 \mathrm{~m} / \mathrm{s}$. 
accessibility, susceptibility to pathology, and relatively large size. ${ }^{26}$ Two other important sites in USE tendon research are the rotator cuff and the common extensor and flexor tendons of the elbow. ${ }^{52-54}$

In Achilles tendinopathy evaluation, the studies showed moderate to perfect correlation between the USE and Bmode US and excellent correlation with functional scores. ${ }^{54,55}$ Coombes et al reported that low shear wave velocity is correlated with higher age, self-reported pain, and with decreased loading capacity of the Achilles tendon. ${ }^{56}$ Slane et al also showed lowering shear wave speed in older adults and reported that aging alters spatial variations in the Achilles tendon. ${ }^{57}$ Studies in athletes demonstrated that tendon softening may predict pain and tendinopathy. ${ }^{55}$ Thus USE may prove useful in the detection of subclinical tendinopathy, and timely preventive measures may be undertaken. ${ }^{55}$ Subclinical changes of Achilles tendon were also shown with USE in patients with ankylosing spondylitis, acromegaly, and in patients with diabetic ulcers. 55

USE has been used to evaluate supraspinatus tendon and muscle, with tendinopathy predominantly presenting with tendon softening but also with regions with increased stiffness (- Fig. 8). ${ }^{55}$ Correlation between USE and Bmode US in the evaluation of rotator cuff was reported. ${ }^{52,53}$ Rosskopf et al showed decreased shear wave velocity with increasing fat content and increasing shear wave velocity in the final stage of fatty infiltration of the supraspinatus muscle. ${ }^{52}$ Seo et al reported that USE is able to quantify the severity of fatty atrophy of the supraspinatus muscle

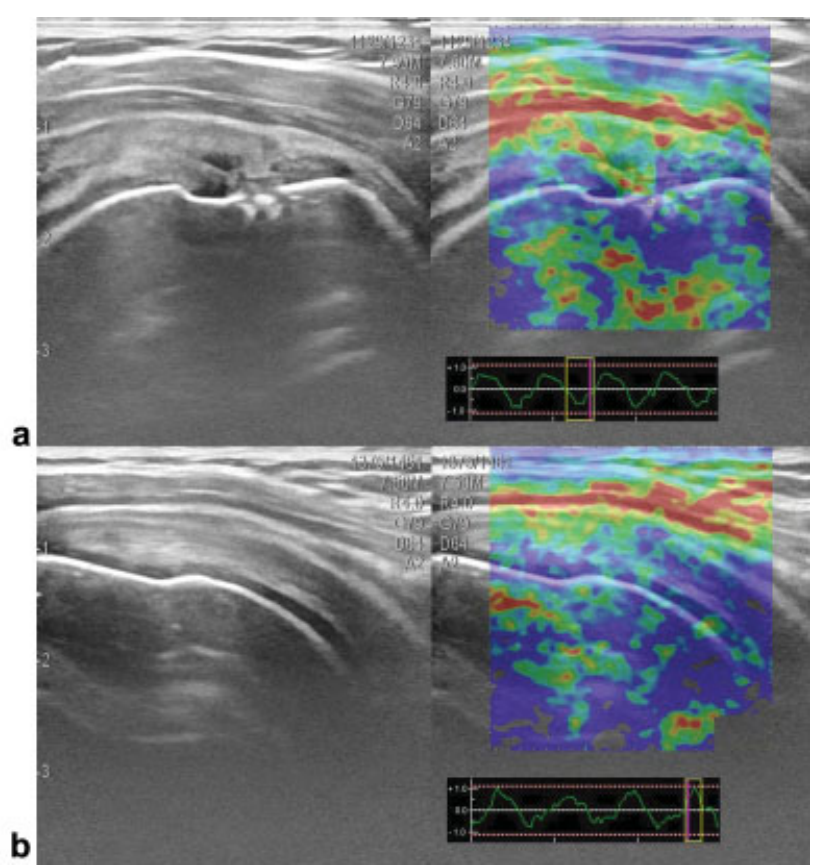

Fig. 8 B-mode and elastogram image of the bilateral supraspinatus tendon in a patient with pain in the right shoulder. Panel a: Articular surface partialthickness tear of the right supraspinatus tendon is seen on the B-mode with heterogeneous elastogram in the corresponding area showing tendon softening (red areas). Panel b: Left shoulder of the same patient showing intact supraspinatus tendon with normal hard (blue) elastogram. The compression cycles may be appreciated. with excellent accuracy and interobserver reliability. ${ }^{53}$ USE may prove to be useful in the preoperative setting in patient selection because it can reflect tendon quality or surgical outcome. $^{58}$

In diagnosing medial epicondylitis, the addition of USE to B-mode US provides a significant improvement in the agreement between imaging and histologic results compared with either B-mode US or USE alone. ${ }^{59}$ Furthermore, USE proved to have higher diagnostic performance compared with B-mode US. ${ }^{60}$ Several studies showed softening of common extensor origin in the patients with lateral epicondylitis. $^{55}$

B-mode US or USE can depict fatty infiltration, loss of collagen structure, and capillary proliferation; however, USE may be able to show loss of fiber integrity with greater sensitivity than B-mode US. ${ }^{54}$ The degenerative sign of loss of fiber integrity with mucoid degeneration is associated with pathologic softening on USE. ${ }^{26,54}$ Most studies confirmed this as the predominant pattern of tendinopathy; however, few studies showed increased tendon stiffness in tendinopathy. ${ }^{26}$ Patients with surgical repair of complete Achilles tendon rupture showed a harder and heterogeneous pattern compared with healthy controls, indicating the difference of reparative compared with normal tendon tissue. ${ }^{61}$ Furthermore, in a 1-year follow-up of the postsurgical elasticity values of the Achilles tendon, SWE showed good correlation with functional outcomes. ${ }^{62}$ These findings are supported by a laboratory study on transected Achilles tendons in rabbits that showed progressive hardening with healing time, a finding supported with histologic correlation. ${ }^{63}$ A general statement on the elastic properties in tendinopathy may not be straightforward, and perhaps we should aim at identifying areas of a heterogeneous distorted elastogram (hardening or softening) as an indicator of tendinopathy. ${ }^{26}$

\section{Ligaments}

The literature on USE for imaging ligaments is scarce. ${ }^{64-67}$ Joint positioning plays an important role in ligament USE imaging because stretched ligament or relaxed ligament differ substantially. ${ }^{64}$ Two articles studied healthy volunteers for the characterization of the anterior talofibular ligament and collateral ligament of the elbow. ${ }^{65,66}$ Both concluded that USE could prove useful in evaluation of a ligament injury and in the follow-up of the healing process. ${ }^{65,66}$ Gupta et al showed no significant differences in shear wave velocity between the dominant and nondominant arm when examining the ulnar collateral ligament. ${ }^{65}$ A similar observation was made by Wu et al when examining the coracohumeral ligament in healthy volunteers (-Fig. 9) ${ }^{64}$ Furthermore, the same study showed a stiffer coracohumeral ligament in patients with adhesive capsulitis of the shoulder. ${ }^{64}$ Interestingly, Mhanna et al showed significantly increased shear wave velocity of the traverse carpal ligament in pianists relative to non-pianists and proposed that ligament maladaptation may explain why populations with repetitive hand movements are at an increased risk of developing MSK pathologies. ${ }^{67}$ 


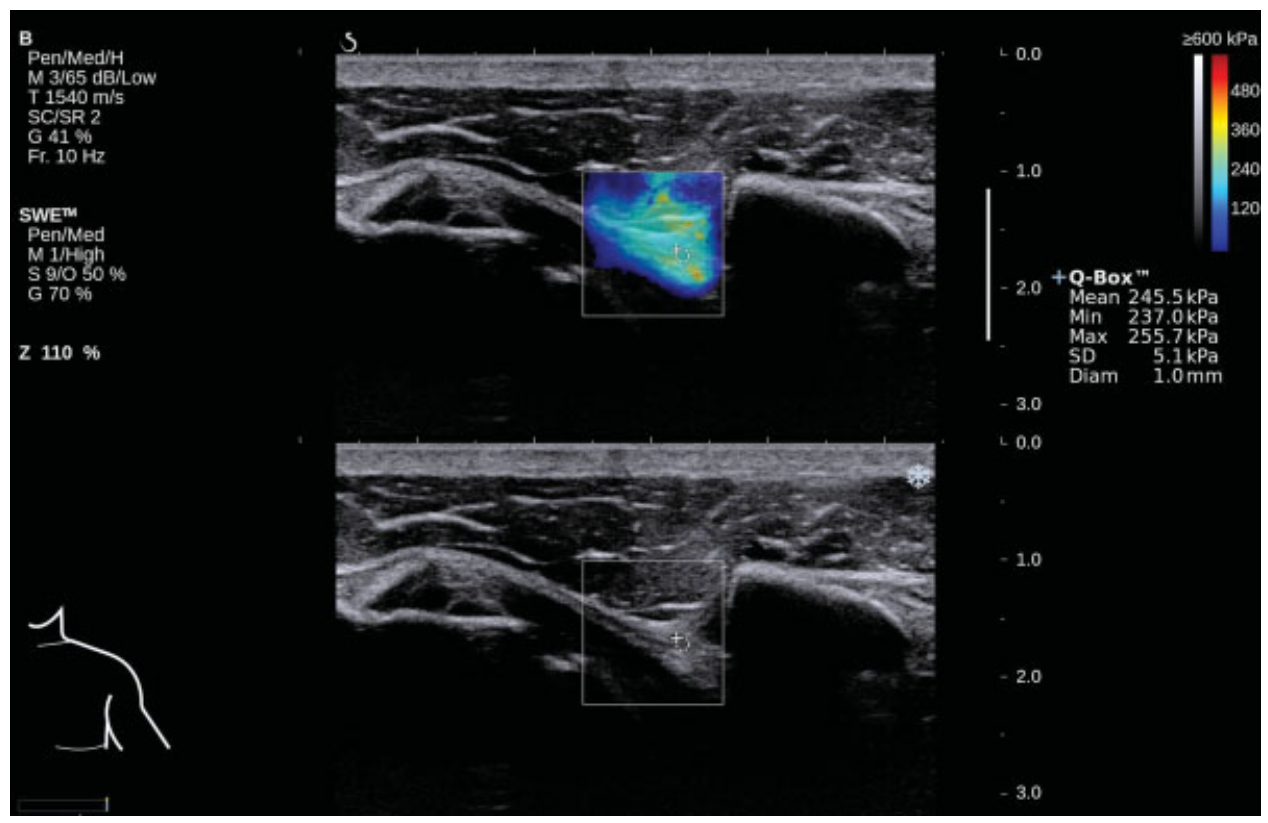

Fig. 9 Shear wave elastogram of the coracohumeral ligament in the shoulder neutral position in a 41-year-old man with adhesive capsulitis of the shoulder. The shear modulus within the region of interest was $245.5 \mathrm{kPa}$.

\section{Technical Considerations}

The presence of various commercially available systems, imaging methods, and techniques make USE homogenization difficult. Certain technical considerations must be recognized by the user because standardization of USE technical settings will establish accuracy and thus identify variations over time that may be recognized as true physiologic changes. ${ }^{67}$ Kot et al recommended that SWE of the tendon tendon and muscle should be performed with the lightest transducer pressure and a shorter acquisition time for SWE while measuring the mean elastic modulus regardless of ROI size. ${ }^{68}$ It must be noted that USE values depend on the surrounding tissues and depth. ${ }^{69}$ Bone vicinity especially needs to be considered because it decreases the shear wave velocity of the overlying tissues. ${ }^{69}$

Different transducer positioning has been used across studies (transverse versus longitudinal position). ${ }^{34}$ The shear waves propagate faster in contracted tendons and muscles and along the long axis of tendons. The transducer must be oriented longitudinally to the muscle fibers to achieve accurate and reliable SWE measurements. ${ }^{70}$

$\mathrm{CE}$ is an operator-dependent technique, with a recommendation to record several (at least three) compression-relaxation cycles as cine loops, and the best elastograms should be selected for evaluation. ${ }^{70}$ The transducer should be perpendicular to the tissue to avoid anisotropy because the B-mode US appearance influences the quality of the elastogram. ${ }^{70}$ The elastogram is not influenced by the use of standoff devices in imaging superficial structures because a minimum distance of $3 \mathrm{~mm}$ is needed between transducer and target lesion. ${ }^{70}$

The Quantitative Imaging Biomarkers Alliance (QIBA) was created by the Radiological Society of North America to establish guidelines on quantitative imaging and the use of imaging biomarkers in clinical practice. QIBA standardized methods on US phantoms to understand the sources of bias and variance in SWE. ${ }^{70}$ They found significant differences in shear wave speed estimation among systems and according to the depth of the object. ${ }^{71}$ Important guidelines and recommendations on elastography were created by the European Federation of Societies for Ultrasound in Medicine and Biology (EFSUMB). ${ }^{3,70}$

Standardization in reporting USE measurements is important. When the formula to calculate Young's modulus is used, the tissue density is assumed to be constant. This assumption is correct for isotropic tissues such as the liver or thyroid gland, but the soft tissues examined in the MSK system are heterogenous and anisotropic. Thus SWE in the MSK system should be reported as shear wave velocity $(\mathrm{m} / \mathrm{sec})$ rather than in shear modulus $(\mathrm{kPa}) .^{10}$

\section{Future Directions}

Although significant work and important advances in USE have been made, further efforts are needed to define its various potential applications and to assure its progress. Despite great interest, the literature is still limited to small population studies without control groups, utilizing different techniques and evaluation methods. Multicenter long-term controlled studies are needed with large populations of different ages and levels of activity with long-term follow-up in correlation with other imaging methods and clinical data. ${ }^{2}$ Considerable action should be taken in the standardization of various technical settings before obtaining valid data for diagnosis and guiding corrective therapy. In this direction, initiatives made by QIBA and EFSUMB are of great importance. However, this may not be sufficient. We should aim to close collaboration between industry and clinical researchers to make optimized 
protocols dedicated to MSK applications. ${ }^{2}$ Furthermore, USE is currently equipped in high-end US machines and would be helpful in wider applications as a bedside clinical tool.

Software for image fusion is integrated in some USE machines, offering fusion of cross-sectional imaging methods and real-time USE. With cross-referencing imaging methods, USE may yield similar information as those provided by MRI or $\mathrm{CT}^{7}{ }^{\text {Thee-dimensional (3D) images have }}$ been reconstructed from two-dimensional elastograms. ${ }^{7}$ The potential use of real-time 3D elastographic analysis would prove useful in the MSK field in the evaluation of mass lesions in preoperative planning and follow-up. ${ }^{7}$

\section{Conclusion}

USE is a tool for the imaging of MSK soft tissue, providing biomechanical data along with the morphological data supplied by a conventional US examination, thus allowing an insight into the biomechanics of normal or diseased tissue in the clinical setting. The various USE techniques available for clinical use create diversity in the reported data and necessitate thorough knowledge of the physics, the limitations, and the techniques of application in various tissues. At present, USE is an additional tool to current standard practice in MSK imaging. The evidence supporting the role of USE for assessing MSK soft tissue is constantly increasing in the literature; however, more work is needed to ensure validation of the findings and assessment of their clinical relevance.

\section{Conflict of Interest \\ None declared.}

\section{References}

1 Sigrist RMS, Liau J, Kaffas AE, Chammas MC, Willmann JK. Ultrasound elastography: review of techniques and clinical applications. Theranostics 2017;7(05):1303-1329

2 Drakonaki EE, Allen GM, Wilson DJ. Ultrasound elastography for musculoskeletal applications. Br J Radiol 2012;85(1019):1435-1445

3 Bamber J, Cosgrove D, Dietrich CF, et al. EFSUMB guidelines and recommendations on the clinical use of ultrasound elastography. Part 1: Basic principles and technology. Ultraschall Med 2013;34 (02):169-184

4 Kane D, Grassi W, Sturrock R, Balint PV. A brief history of musculoskeletal ultrasound: 'From bats and ships to babies and hips.'. Rheumatology (Oxford) 2004;43(07):931-933

5 Mariappan YK, Glaser KJ, Ehman RL. Magnetic resonance elastography: a review. Clin Anat 2010;23(05):497-511

6 Sconfienza LM, Albano D, Allen G, et al. Clinical indications for musculoskeletal ultrasound updated in 2017 by European Society of Musculoskeletal Radiology (ESSR) consensus. Eur Radiol 2018; 28(12):5338-5351

7 Klauser AS, Miyamoto H, Bellmann-Weiler R, Feuchtner GM, Wick MC, Jaschke WR. Sonoelastography: musculoskeletal applications. Radiology 2014;272(03):622-633

$8 \mathrm{Wu}$ CH, Wang TG. Author reply to "color scaling in sonoelastography.". Am J Phys Med Rehabil 2015;94(09):e85

9 Wu CH, Chen WS, Park GY, Wang TG, Lew HL. Musculoskeletal sonoelastography: a focused review of its diagnostic applications for evaluating tendons and fascia. J Med Ultrasound 2012;20:79-86

10 Urban MW, Nenadic IZ, Chen S, Greenleaf JF. Discrepancies in reporting tissue material properties. J Ultrasound Med 2013;32 (05):886-888
11 Sconfienza LM, Silvestri E, Orlandi D, et al. Real-time sonoelastography of the plantar fascia: comparison between patients with plantar fasciitis and healthy control subjects. Radiology 2013;267 (01):195-200

12 Langevin HM, Fox JR, Koptiuch C, et al. Reduced thoracolumbar fascia shear strain in human chronic low back pain. BMC Musculoskelet Disord 2011;12:203

13 Luomala T, Pihlman M, Heiskanen J, Stecco C. Case study: could ultrasound and elastography visualized densified areas inside the deep fascia? J Bodyw Mov Ther 2014;18(03):462-468

$14 \mathrm{Wu} \mathrm{CH}$, Chen WS, Wang TG, Lew HL. Can sonoelastography detect plantar fasciitis earlier than traditional B-mode ultrasonography? Am J Phys Med Rehabil 2012;91(02):185

$15 \mathrm{Wu} \mathrm{CH}$, Chen WS, Wang TG. Plantar fascia softening in plantar fasciitis with normal B-mode sonography. Skeletal Radiol 2015; 44(11):1603-1607

$16 \mathrm{Wu} \mathrm{CH}$, Chang KV, Mio S, Chen WS, Wang TG. Sonoelastography of the plantar fascia. Radiology 2011;259(02):502-507

17 Wee TC, Simon NG. Ultrasound elastography for the evaluation of peripheral nerves: a systematic review. Muscle Nerve 2019;60 (05):501-512

18 Zhu B, Yan F, He Y, et al. Evaluation of the healthy median nerve elasticity: feasibility and reliability of shear wave elastography. Medicine (Baltimore) 2018;97(43):e12956

19 Paluch Ł, Pietruski P, Walecki J, Noszczyk BH. Wrist to forearm ratio as a median nerve shear wave elastography test in carpal tunnel syndrome diagnosis. J Plast Reconstr Aesthet Surg 2018;71 (08):1146-1152

20 Yoshii Y, Tung WL, Ishii T. Strain and morphological changes of median nerve after carpal tunnel release. J Ultrasound Med 2017; 36(06):1153-1159

21 Klauser AS, Miyamoto H, Martinoli C, et al. Sonoelastographic findings of carpal tunnel injection. Ultraschall Med 2015;36(06): 618-622

22 Dikici AS, Ustabasioglu FE, Delil S, et al. Evaluation of the tibial nerve with shear-wave elastography: a potential sonographic method for the diagnosis of diabetic peripheral neuropathy. Radiology 2017;282(02):494-501

23 Sconfienza LM, Adriaensen M, Albano D, et al. Clinical indications for image-guided interventional procedures in the musculoskeletal system: a Delphi-based consensus paper from the European Society of Musculoskeletal Radiology (ESSR)-part I, shoulder. Eur Radiol 2019; September 16 (Epub ahead of print)

24 Sconfienza LM, Adriaensen M, Albano D, et al; Ultrasound and Interventional Subcommittees of the European Society of Musculoskeletal Radiology (ESSR). Clinical indications for image guided interventional procedures in the musculoskeletal system: a Delphi-based consensus paper from the European Society of Musculoskeletal Radiology (ESSR)-part III, nerves of the upper limb. Eur Radiol 2019; November 11 (Epub ahead of print)

25 Sconfienza LM, Adriaensen M, Albano D, et al. Clinical indications for image-guided interventional procedures in the musculoskeletal system: a Delphi-based consensus paper from the European Society of Musculoskeletal Radiology (ESSR)-part II, elbow and wrist. Eur Radiol 2019;(Epub ahead of print)

26 Winn N, Lalam R, Cassar-Pullicino V. Sonoelastography in the musculoskeletal system: current role and future directions. World J Radiol 2016;8(11):868-879

27 Hahn S, Lee YH, Lee SH, Suh JS. Value of the strain ratio on ultrasonic elastography for differentiation of benign and malignant soft tissue tumors. J Ultrasound Med 2017;36(01):121-127

28 Pass B, Jafari M, Rowbotham E, Hensor EM, Gupta H, Robinson P. Do quantitative and qualitative shear wave elastography have a role in evaluating musculoskeletal soft tissue masses? Eur Radiol 2017;27(02):723-731

29 Riishede I, Ewertsen C, Carlsen J, Petersen MM, Jensen F, Nielsen MB. Strain elastography for prediction of malignancy in soft tissue tumours-preliminary results. Ultraschall Med 2015;36(04):369-374 
30 Lalitha P, Reddy MCh, Reddy KJ. Musculoskeletal applications of elastography: a pictorial essay of our initial experience. Korean J Radiol 2011;12(03):365-375

31 Madej T, Flak-Nurzyńska J, Dutkiewicz E, Ciechomska A, Kowalczyk J, Wieczorek AP. Ultrasound image of malignant bone tumors in children. An analysis of nine patients diagnosed in 2011-2016. J Ultrason 2018;18(73):103-111

32 Harmon B, Wells M, Park D, Gao J. Ultrasound elastography in neuromuscular and movement disorders. Clin Imaging 2019; 53:35-42

33 Creze M, Nordez A, Soubeyrand M, Rocher L, Maître X, Bellin MF. Shear wave sonoelastography of skeletal muscle: basic principles, biomechanical concepts, clinical applications, and future perspectives. Skeletal Radiol 2018;47(04):457-471

34 Mendes B, Firmino T, Oliveira R, et al. Hamstring stiffness pattern during contraction in healthy individuals: analysis by ultrasoundbased shear wave elastography. Eur J Appl Physiol 2018;118(11): 2403-2415

35 Koppenhaver SL, Scutella D, Sorrell BA, et al. Normative parameters and anthropometric variability of lumbar muscle stiffness using ultrasound shear-wave elastography. Clin Biomech (Bristol, Avon) 2019;62:113-120

36 Chino K, Takahashi H. Measurement of gastrocnemius muscle elasticity by shear wave elastography: association with passive ankle joint stiffness and sex differences. Eur J Appl Physiol 2016; 116(04):823-830

$37 \mathrm{Wu} \mathrm{CH}$. Does sex influence biceps brachii muscle stiffness? Clin Imaging 2018;49:198

38 Alfuraih AM, Tan AL, O'Connor P, Emery P, Wakefield RJ. The effect of ageing on shear wave elastography muscle stiffness in adults. Aging Clin Exp Res 2019;31(12):1755-1763

39 Akagi R, Fukui T, Kubota M, Nakamura M, Ema R. Muscle shear moduli changes and frequency of alternate muscle activity of plantar flexor synergists induced by prolonged low-level contraction. Front Physiol 2017;8:708

40 Saito A, Wakasa M, Kimoto M, et al. Age-related changes in muscle elasticity and thickness of the lower extremities are associated with physical functions among community-dwelling older women. Geriatr Gerontol Int 2019;19(01):61-65

41 Yanagisawa O, Niitsu M, Kurihara T, Fukubayashi T. Evaluation of human muscle hardness after dynamic exercise with ultrasound real-time tissue elastography: a feasibility study. Clin Radiol 2011;66(09):815-819

42 Agten CA, Buck FM, Dyer L, Flück M, Pfirrmann CW, Rosskopf AB. Delayed-onset muscle soreness: temporal assessment with quantitative MRI and shear-wave ultrasound elastography. AJR Am J Roentgenol 2017;208(02):402-412

43 Sikdar S, Shah JP, Gebreab T, et al. Novel applications of ultrasound technology to visualize and characterize myofascial trigger points and surrounding soft tissue. Arch Phys Med Rehabil 2009;90(11): 1829-1838

44 Botar Jid C, Vasilescu D, Damian L, Dumitriu D, Ciurea A, Dudea SM. Musculoskeletal sonoelastography. Pictorial essay. Med Ultrason 2012;14(03):239-245

45 Lv F, Tang J, Luo Y, et al. Muscle crush injury of extremity: quantitative elastography with supersonic shear imaging. Ultrasound Med Biol 2012;38(05):795-802

46 Botar-Jid C, Damian L, Dudea SM, Vasilescu D, Rednic S, Badea R. The contribution of ultrasonography and sonoelastography in assessment of myositis. Med Ultrason 2010;12(02):120-126

47 Lee SS, Gaebler-Spira D, Zhang LQ, Rymer WZ, Steele KM. Use of shear wave ultrasound elastography to quantify muscle properties in cerebral palsy. Clin Biomech (Bristol, Avon) 2016; $31: 20-28$

$48 \mathrm{Wu} \mathrm{CH}$, Ho YC, Hsiao MY, Chen WS, Wang TG. Evaluation of post-stroke spastic muscle stiffness using shear wave ultrasound elastography. Ultrasound Med Biol 2017;43(06):11051111
49 Illomei G, Spinicci G, Locci E, Marrosu MG. Muscle elastography: a new imaging technique for multiple sclerosis spasticity measurement. Neurol Sci 2017;38(03):433-439

$50 \mathrm{Wu}$ CH, Wang TG. Measurement of muscle stiffness in children with spastic cerebral palsy. Radiology 2012;265(02):647; author reply 647-648

$51 \mathrm{Wu} \mathrm{CH}$. A few considerations on "Muscle material properties in passive and active stroke-impaired muscle.". J Biomech 2019; 93:231

52 Rosskopf AB, Ehrmann C, Buck FM, Gerber C, Flück M, Pfirrmann CW. Quantitative shear-wave US elastography of the supraspinatus muscle: reliability of the method and relation to tendon integrity and muscle quality. Radiology 2016;278(02):465-474

53 Seo JB, Yoo JS, Ryu JW. The accuracy of sonoelastography in fatty degeneration of the supraspinatus: a comparison of magnetic resonance imaging and conventional ultrasonography. J Ultrasound 2014;17(04):279-285

54 Klauser AS, Miyamoto H, Tamegger M, et al. Achilles tendon assessed with sonoelastography: histologic agreement. Radiology 2013;267(03):837-842

55 Prado-Costa R, Rebelo J, Monteiro-Barroso J, Preto AS. Ultrasound elastography: compression elastography and shear-wave elastography in the assessment of tendon injury. Insights Imaging 2018;9 (05):791-814

56 Coombes BK, Tucker K, Vicenzino B, et al. Achilles and patellar tendinopathy display opposite changes in elastic properties: a shear wave elastography study. Scand J Med Sci Sports 2018;28 (03):1201-1208

57 Slane LC, Martin J, DeWall R, Thelen D, Lee K. Quantitative ultrasound mapping of regional variations in shear wave speeds of the aging Achilles tendon. Eur Radiol 2017;27(02):474-482

58 Hou SW, Merkle AN, Babb JS, McCabe R, Gyftopoulos S, Adler RS. Shear wave ultrasound elastographic evaluation of the rotator cuff tendon. J Ultrasound Med 2017;36(01):95-106

59 Klauser AS, Pamminger MJ, Halpern EJ, et al. Sonoelastography of the common flexor tendon of the elbow with histologic agreement: a cadaveric study. Radiology 2017;283(02): 486-491

60 Shin M, Hahn S, Yi J, Lim YJ, Bang JY. Clinical application of realtime sonoelastography for evaluation of medial epicondylitis: a pilot study. Ultrasound Med Biol 2019;45(01):246-254

61 Tan S, Kudaş S, Özcan AS, et al. Real-time sonoelastography of the Achilles tendon: pattern description in healthy subjects and patients with surgically repaired complete ruptures. Skeletal Radiol 2012;41(09):1067-1072

62 Zhang LN, Wan WB, Wang YX, et al. Evaluation of elastic stiffness in healing Achilles tendon after surgical repair of a tendon rupture using in vivo ultrasound shear wave elastography. Med Sci Monit 2016;22:1186-1191

63 Yamamoto Y, Yamaguchi S, Sasho T, et al. Quantitative US elastography can be used to quantify mechanical and histologic tendon healing in a rabbit model of Achilles tendon transection. Radiology 2017;283(02):408-417

$64 \mathrm{Wu} \mathrm{CH}$, Chen WS, Wang TG. Elasticity of the coracohumeral ligament in patients with adhesive capsulitis of the shoulder. Radiology 2016;278(02):458-464

65 Gupta N, Labis JS, Harris J, et al. Shear-wave elastography of the ulnar collateral ligament of the elbow in healthy volunteers: a pilot study. Skeletal Radiol 2019;48(08):1241-1249

66 Hotfiel T, Heiss R, Janka R, et al. Acoustic radiation force impulse tissue characterization of the anterior talofibular ligament: a promising noninvasive approach in ankle imaging. Phys Sportsmed 2018;46(04):435-440

67 Mhanna C, Marquardt TL, Li ZM. Adaptation of the transverse carpal ligament associated with repetitive hand use in pianists. PLOS One 2016;11(03):e0150174

68 Kot BC, Zhang ZJ, Lee AW, Leung VY, Fu SN. Elastic modulus of muscle and tendon with shear wave ultrasound elastography: 
166 Ultrasound Elastography in MSK Radiology Snoj et al.

variations with different technical settings. PLOS One 2012;7(08): e44348

69 Ewertsen C, Carlsen JF, Christiansen IR, Jensen JA, Nielsen MB. Evaluation of healthy muscle tissue by strain and shear wave elastography-dependency on depth and ROI position in relation to underlying bone. Ultrasonics 2016;71:127-133
70 Săftoiu A, Gilja OH, Sidhu PS, et al. The EFSUMB Guidelines and Recommendations for the clinical practice of elastography in nonhepatic applications: update 2018. Ultraschall Med 2019;40(04): 425-453

71 Ryu J, Jeong WK. Current status of musculoskeletal application of shear wave elastography. Ultrasonography 2017;36(03):185-197 